


\title{
Neuroeducational Intervention in Children with Intellectual Disabilities - Intervención Neuroeducativa en Niñoscon Discapacidad Intelectual
}

\author{
Yesica Milena Flórez Bautista \\ Silvia González Hartmann \\ Daniela Celia Graiño \\ Mónica Alejandra Machaädo Sánchez \\ Jessica Marcela Arevalo Parra
}

\begin{abstract}
An adequate intervention in the classroom for children with intellectual disabilities has been one of the main concerns of the teaching team, due to the high dropout rate that is currently occurring in Colombia (MEN, 2015). The lack of knowledge on how to intervene is one of the most influential reasons for children in condition of intellectual disability to fail to integrate successfully, nor to achieve an optimal learning process, these factors generate frustration not only in the student, but in teachers and family members, (Martínez, 2017) therefore, the need to investigate the impact caused by different strategies in the learning process in children with intellectual disability emerged. After a review of academic literature, it was found that neuroeducation provides the necessary elements that children with disabilities need so that their process in the classroom is not truncated.
\end{abstract}

The purpose of this research, therefore, is to propose innovative, inclusive and participatory neuroeducational strategies for: students with disabilities, teachers who train inclusive and integrative processes, and the family as the first link of contact and experience. These strategies consist of the union and exploration of practices, procedures and knowledge about neuroscience and education, recognizing the importance of brain mechanisms in the construction of cognitive, socio-affective and adaptive processes. These strategies are proposed in an educational world that is still conditioned by access barriers that limit and maintain the vision of disability as a handicap, and not as a condition of life.

Key Words

Intellectual disability, neuroeducation, inclusion, educational strategies 


\section{Resumen}

Una intervención adecuada dentro del aula en niños con discapacidad intelectual ha sido una de las principales preocupaciones del equipo docente, debido al alto índice de deserción escolar que se está presentando actualmente en Colombia, (MEN, 2015). La falta de conocimiento sobre cómo intervenir es una de las razones más influyentes para que los niños en condición de discapacidad intelectual no logren integrarse de manera exitosa, ni alcanzar un proceso óptimo de aprendizaje, estos factores generan una frustración no solo en el estudiante, sino en los docentes y familiares, (Martínez, 2017) por lo cual, emergió la necesidad de investigar sobre el impacto que ocasionan diferentes estrategias en el proceso de aprendizaje en niños con discapacidad intelectual. Después de una revisión de literatura académica se encontró que la neuroeducación aporta los elementos necesarios que el niño con discapacidad necesita para que su proceso en el aula no se vea truncado.

La finalidad por tanto de la presente investigación, consiste en proponer estrategias neuroeducativas innovadoras, incluyentes y participativas para: los estudiantes en condición de discapacidad, los docentes formadores de procesos inclusivos e integradores y la familia como primer vínculo de contacto y experiencia. Estas estrategias que consisten en la unión y exploración de prácticas, procedimientos y conocimientos sobre la neurociencia y la educación, reconocen la importancia de los mecanismos cerebrales en la construcción de procesos cognitivos, socio afectivos y de adaptación. Estas estrategias se proponen en un mundo educativo que aún se ve condicionado por barreras de acceso que limitan y mantienen la visión de la discapacidad desde la minusvalía, y no como una condición de vida.

\section{Palabras clave}

Discapacidad intelectual, neuroeducación, inclusión, estrategias educativas 


\section{Introducción}

En el año 2006 la ONU definió la discapacidad como un "concepto que evoluciona y que resulta de la interacción entre las personas con deficiencias y las barreras debidas a la actitud y al entorno que evitan su participación plena y efectiva en la sociedad, en igualdad de condiciones "ONU" (2006).

Dentro de este grupo se encuentran a las personas con Discapacidad Intelectual que, a nivel global según HUANG (2016), afecta al $2 \%$ de la población mundial, sin embargo, a nivel nacional la situación es más compleja ya que según los últimos datos obtenidos en Colombia por medio del Censo General del 2005, un porcentaje de $6,9 \%$ de la población posee un déficit intelectual, lo que hace pensar que actualmente este número es aún mayor, como se sugiere más adelante.

Según lo expuesto anteriormente se evidencia que la Discapacidad Intelectual en el aula escolar supone un reto tanto para los docentes como para los alumnos, si bien el estudiante con discapacidad intelectual tiene un ritmo más lento para aprender y requiere de un mayor número de experiencias para comprender, y asimilar nuevos conceptos, no quiere que el aprendizaje en niños con esta condición sea un reto imposible de alcanzar, sin embargo, es muy importante tener un amplio conocimiento sobre las condiciones en cuanto a funciones intelectuales que presenta el niño y en base a ello usar las estrategias neuroeducativas más apropiadas, pues aunque haya un mismo diagnóstico sobre discapacidad intelectual, cada niño es diferente. (Martínez 2017).

A través de la intervención neuroeducativa se pretende disminuir el rendimiento escolar insatisfactorio y frustrante para el estudiante que muchas veces no es reconocido dentro de un grupo debido a: déficits sensoriales significativos, no posesión de un lenguaje acorde a la edad y con implicaciones neurológicas notorias. (AAIDD, 2020). Cabe resaltar que no solamente el ámbito escolar se ve afectado, el niño con condición de discapacidad intelectual presenta limitaciones en su diario vivir, pues su independencia puede verse afectada, así como la capacidad de desenvolverse en diferentes contextos. (Alvarado, 2016).

Si bien en Colombia se ha adoptado la educación inclusiva mediante el decreto 1421 de 2017, (Congreso de la República) las cifras de deserción escolar en la población con discapacidad intelectual aún siguen siendo altas, según el MEN 
(2015) es del 10\%, perjudicando de esta manera el desarrollo normal del proceso educativo. (Fuentes, 2013).

Según lo anterior, surge entonces la siguiente pregunta: ¿Cuál es la principal falla en este proceso que provoca el nivel tan alto de deserción en este grupo poblacional? Este fenómeno trae consecuencias, no solo durante la niñez, sino durante el resto de su trayectoria de vida. La respuesta, sin duda guarda una directa relación con las prácticas pedagógicas relacionadas a la neuroeducación, consecuencia de la falta de conocimiento de los docentes, acerca de las estrategias de estimulación que estos niños requieren en el aula.

Es a partir de la necesidad que se presenta en el aula con los niños, con discapacidad intelectual, que se considera que la neuroeducación puede dar una buena respuesta a esta problemática, pues esta disciplina tiene como función: estudiar el cerebro durante el proceso de enseñanza -aprendizaje, analizar el desarrollo del mismo, y su reacción a los estímulos que posteriormente se constituyen en los conocimientos. (Fernández, 2019).

\section{Justificación}

Según la Organización mundial de la Salud (2020), el 15\% de la población mundial padece de algún tipo de discapacidad intelectual, en países en vía de desarrollo como es el caso de Colombia, la discapacidad intelectual es un trastorno que tiene mayor prevalencia, factores de tipo económico y sociodemográficos; el sistema de salud influye en el desarrollo cognitivo del individuo (Mercurio, 2016). Se ha Registrado la Localización y Caracterización de Personas con Discapacidad RLCPD por parte del Ministerio de Salud y Protección social de Colombia (2018) reconociéndose que en Colombia hay 1.418 .065 de personas con dicha condición, por esta razón se observa una mayor necesidad de dar prioridad a las personas con Discapacidad Intelectual y más si se tiene en cuenta que de la cifra mencionada anteriormente, un tercio, no asiste a establecimientos educativos y tan solo el $16 \%$ de ellos han alcanzado la básica primaria. (MEN, 2015)

A partir de lo mencionado anteriormente, en cualquier circunstancia en la que se presenten dificultades del aprendizaje en un niño, se debe observar su capacidad de interacción con el programa, es decir, las dificultades que presenta el niño por su diagnóstico, como las que se puede generar a partir de las estrategias de educación utilizadas. (Gallegos y Rodríguez, 2013). 
Con base en lo anterior, se entiende que el aula escolar es crucial para satisfacer esas necesidades, en la cual se debe llevar a cabo cualquier ajuste en el caso que se requiera. Para ello es necesario que los docentes estén preparados para intervenir en el proceso de aprendizaje del niño en condición de discapacidad, sin embargo, Saeed (2014) afirma lo siguiente: "teniendo en cuenta la naturaleza divergente de la población con Discapacidad intelectual, todavía falta investigación en esta área hasta la fecha por lo que un aporte en este ámbito es crucial para el conocimiento científico y para esta población”, quienes su calidad de vida y la de sus familiares se ve afectada de manera significativa, por lo tanto el propósito de este proyecto de investigación es comprender el impacto que genera las diferentes estrategias neuroeducativas en niños con discapacidad intelectual según el hallazgo obtenido a partir de diferentes autores.

Adicional a lo anterior Gómez, Verdugo \& Arias (2010) señalan que el campo educativo donde se promueva la neuroeducación es fundamental para el desarrollo integral del niño en condición de discapacidad, dado que la neuroeducación le aporta al maestro un mayor conocimiento acerca de las funciones cerebrales y de los factores que ejercen influencia durante su crecimiento, desarrollo y funcionalidad. Desde el momento en que la educación comienza a incorporar a las neurociencias, éstas le ofrecen la posibilidad de comprender los conocimientos básicos acerca de las bases neurales del aprendizaje, la memoria, las emociones y demás funciones cerebrales que son estimuladas y fortalecidas constantemente en el aula. Esta incorporación resulta fundamental para mejorar los procesos de aprendizaje del aula en general y más aún los procesos de aquellos niños con discapacidades cognitivas, ya que ayudan a potenciar sus capacidades neurocognitivas y emocionales de la mano de los profesores referentes directos. (Rojas, 2019).

\section{Marco Teórico}

Como paso previo al desarrollo del estudio práctico, se considera necesario hacer referencia a los conceptos de discapacidad intelectual y neuroeducación existentes en la literatura académica que abordan esta problemática.

\section{Acerca de la discapacidad intelectual y su condición de vida}

El término discapacidad intelectual (DI) es entendido como la adquisición lenta e incompleta de las habilidades cognitivas durante el desarrollo humano. Alvarado (2016); Lo cual implica entre otras cosas, que la persona presente dificultades para comprender, aprender y desarrollar habilidades adaptativas, analíticas y 
psicosociales según su periodo evolutivo, evidenciando así, diversas limitaciones en su diario vivir (Seda, 2021). En consecuencia en la Discapacidad intelectual, la calidad de vida e independencia se ve condicionada en diferentes contextos, uno de ellos y de vital importancia es en el sistema educativo, el cual requiere estrategias de educación que permitan un aprendizaje incluyente y participativo. (Caballero, 2018).

Según el Manual Diagnóstico y Estadístico de los trastornos mentales DSM-V de la American Psychological Association (2014) la discapacidad intelectual se encuentra en la categoría de los trastornos del neurodesarrollo, donde se destacan descriptores principales que categorizan diversas alteraciones cognitivas en la atención, planificación, velocidad de procesamiento, organización y adaptación de la conducta, generando así una condición asociada a delimitar la autonomía y responsabilidad de labores propias a sistemas externos que en muchas ocasiones restringen la cooperación social. (Hurtado y Agudelo, 2014).

El lugar donde se presentan los mayores problemas para los niños en condición de discapacidad intelectual es el aula escolar; donde se evidencia una deficiente intervención por parte de los maestros y cuidadores en las experiencia educativas flexibles y acordes a las necesidades de desarrollo psicosocial (Cabrera, Lizarazo \& Medina 2016). Por consiguiente, los criterios diagnósticos y las impresiones impuestas a los estudiantes con discapacidad intelectual han generado una serie de críticas frente a la concepción de cómo realmente se percibe el trastorno, anotando que más allá de los juicios, es necesario la concientización y reestructuración del constructo social y académico, que restringe el desarrollo y la funcionalidad de procesos cognitivos y emocionales. (Alargada y Giménez, 2018).

Está carente intervención cobra una importancia fundamental si se tiene en cuenta que el ámbito escolar para cualquier niño ejerce una enorme influencia en el proceso de desarrollo cerebral. (Caballero, 2018). En promedio el proceso educativo abarca un periodo de tiempo de 14 años y muchas horas dentro de las instituciones educativas. A partir de estos elementos, se considera fundamental que los niños en condición de discapacidad intelectual sean sujetos de excelentes prácticas pedagógicas, donde el conocimiento de esta problemática, por parte de los educadores, permita identificar las habilidades y conocer las estrategias para lograr potenciarlas. (Campos, 2010). Lo que generalmente se presenta es la carencia de los conocimientos necesarios acerca de mejores prácticas pedagógicas por parte de los maestros. (Florez, 2015). 


\section{Neuroeducación, aprendizaje y discapacidad intelectual}

La necesidad de construir ambientes de participación para los estudiantes en condición de discapacidad intelectual, guarda una relación directa con la neuroeducación, una disciplina o práctica educativa que sugiere un encuentro entre la neurociencia y las ciencias de la educación para combatir la exclusión educativa Mora (2017). Convirtiendo el análisis y la interpretación del funcionamiento de las redes neuronales como una estrategia pedagógica, integral e incluyente, en aulas diversas, buscando resignificar aprendizajes, necesidades y adaptaciones, curriculares y psicosociales múltiples en contextos pedagógicos restringidos Figueroa y Farnum (2020).

En otras palabras, la neuroeducación es considerada como el arte de educar a través del funcionamiento y desarrollo evolutivo del cerebro Mora, (2017). Es decir, el objetivo principal de la neuroeducación es reconocer las necesidades sensoriales, cognitivas, neuronales, y psicosociales que son percibidas en los estudiantes, con y sin necesidades educativas especiales, mediante el conocimiento de las estructuras cerebrales involucradas en el proceso de aprendizaje y enseñanza para una educación formativa (Codina, 2015).

\section{Plasticidad cerebral, discapacidad y prácticas educativas.}

Uno de los componentes más relevantes de la neuroeducación es la plasticidad cerebral, entendida ésta como, la capacidad que tiene el cerebro de regenerar neuronas y formar nuevas conexiones sinápticas. Los conocimientos, acerca de la misma y del funcionamiento general del cerebro, son las premisas que la Neuroeducación mantiene para mejorar la práctica pedagógica y el aprendizaje Alagarda y Giménez (2018). De este modo, la plasticidad cerebral permite que el aprendizaje se realice mediante la transformación de la enseñanza, y se reciba en redes neuronales cargadas de información. Sin embargo, el proceso de plasticidad también puede generarse de forma negativa, cuando no se constituyen estrategias duales entre los profesionales de la salud y la comunidad educativa. (Caballero, 2018).

En la discapacidad intelectual la resignificación de las necesidades se sitúa según el proceso de plasticidad y estimulación que se construya, promoviendo calidad de vida independiente e incluyente, eliminado conjeturas, barreras de acceso y construyendo así contextos inclusivos (Flórez, 2015).Por tal razón la familia participa como uno de los principales garantes de los derechos humanos, promoviendo la toma de decisiones frente actividades escolares, que influyen en la 
construcción de personalidad, autonomía y liderazgo, con el fin de edificar su proyecto de vida ( Aguilar, Demóthenes y campos, 2020).

\section{Neuroeducación, familia y procesos pedagógicos en la Discapacidad Intelectual}

La familia constituye nuevos parámetros neuroeducativos para la discapacidad intelectual, reconociendo los estilos, alcances, limitaciones y restricciones de aprendizaje como estrategias para transformar y reconstruir vínculos emocionales, que se encargan de fortalecer los mecanismos de enseñanza (Manjarrez y Hederich, 2020), sin embargo, para los padres y el sistema familiar la asimilación de la DI es un proceso lento, difícil y doloroso según exponen Arellano y Peralta (2015), puesto que los padres construyen expectativas, sueños y deseos propios sobre el proyecto de vida de sus hijos, idealizando un desarrollo de vida basado en sentimientos y sensaciones ajenas a la voluntad propia del niño diverso. (Verdugo, Córdoba y Rodriguez 2020). De igual forma, se crean suposiciones sobre la calidad de vida que se llevará desde una condición de " discapacidad", involucrando miedos y creencias erróneas que identifican la DI como una minusvalía o deficiencia permanente (Contreras, 2015).

El proceso de afrontamiento familiar, se construye desde la emoción hasta la resolución de problemas; es decir, primero los padres o cuidadores reconocen,expresan y redefinen los principios y creencias familiares, luego crean habilidades para desafiar los constructos destructivos de la sociedad, Villavicencio y López (2017). De forma similar, Seda (2021) sostiene: "Los familiares cercanos de una persona con discapacidad tendrán dos obligaciones complementarias, una es la de brindar los cuidados necesarios a quien está en condición de dependencia y otra es la de promover la mayor autonomía posible"

(p.4).

Por consiguiente, es necesario reconocer que la familia necesita entrenamiento, cuidado y psi-coeducación continua sobre los factores biológicos, afectivos, psicológicos y emocionales que se desenvuelven a partir de una impresión diagnóstica, para así, promover una mayor adaptación a las demandas continuas de los contextos en los que se desarrolla el niño con DI (Almario, 2016). Según lo mencionado anteriormente, la familia buscará estrategias novedosas desde diferentes disciplinas, entre ellas la neuroeducación, encargada de promover motivación, autonomía y perseverancia en las destrezas construida mediante 
experiencias socio afectivas, para convivir desde el principio de la oportunidad y equidad en el aula ( Verdugo, Córdoba y Rodriguez 2020).

\section{Neurodiversidad, pedagogía e inclusión educativa}

La educación y la intervención Neuroeducativa comparten el objetivo de innovar y transformar el proceso de pedagogía en un trabajo colaborativo. A través de él se busca el rol activo por parte de estudiantes, docentes y familia. Mediante proyectos transversales que favorezcan procesos sensoriales y experimentales en periodos constantes, incluyentes y participativos Gómez, Muriel y Londoño,(2019). Por consiguiente, los compañeros de aula y los docentes se posicionan como canales de comunicación y transformación que fomentan habilidades de interacción y prácticas imaginativas, que no reconocen diagnósticos, pero promueven la creación de aulas flexibles y dinámicas. Aristizábal, Colorado y Gutiérrez (2016).

La neurociencia necesita instruir a la comunidad educativa sobre los "mecanismos cerebrales", transformando los datos y experiencias pedagógicas, en procesos de aprendizaje completos, eficaces y novedosos, según las necesidades educativas especiales. Howard et al. (2016).

Neuroeducador debe ser consciente de la individualidad de cada aprendizaje, teniendo como premisa que cada cerebro madura a un ritmo diferente. Esto le crea la necesidad de estudiar las alternativas de enseñanza-aprendizaje, y tener la capacidad de mantener una comunicación interdisciplinar, entre el desarrollo permanente en neurociencia, y la experiencia del docente, que a diario aplica sus metodologías. Béjar (2015).

Dado que el desarrollo neurológico es diferente en cada persona, según lo mencionado anteriormente, es importante conocer estrategias neuroeducativas, las cuales son un conjunto de metodologías que se basan en el funcionamiento del cerebro. En esta medida se optimiza el proceso de enseñanza-aprendizaje de cada niño con discapacidad, al promover ambientes dinámicos y enriquecedores. Tacca (2016).

Tabla 1. Estrategias de neuroeducación que favorecen el aprendizaje en niños con discapacidad intelectual 


\begin{tabular}{|c|c|c|}
\hline Autor & \begin{tabular}{|ll} 
Dimensiones & de \\
estrategias & de \\
neuroeducación &
\end{tabular} & $\begin{array}{l}\text { Beneficios en los procesos } \\
\text { cognitivos }\end{array}$ \\
\hline $\begin{array}{l}\text { Maldonado } \\
(2016)\end{array}$ & $\begin{array}{l}\text { Experienciar sensorial, } \\
\text { discapacidad y pedagogía. }\end{array}$ & $\begin{array}{l}\text { Esta metodología permite la } \\
\text { estimulación a la persona con } \\
\text { discapacidad intelectual al convertir } \\
\text { el SNC más maleable para lograr así } \\
\text { la recepción y registro de } \\
\text { información }\end{array}$ \\
\hline $\begin{array}{l}\text { Willingham } \\
\text { (2015) }\end{array}$ & $\begin{array}{l}\text { La plasticidad cerebral } \\
\text { como componente } \\
\text { neuroeducativo, en relación } \\
\text { con la discapacidad } \\
\text { intelectual. }\end{array}$ & $\begin{array}{l}\text { Un niño con discapacidad } \\
\text { intelectual presenta mayor dificultad } \\
\text { en la recepción y registro de } \\
\text { información, sin embargo, cuando } \\
\text { hay un mayor número de } \\
\text { experiencias se desarrolla de mejor } \\
\text { manera la capacidad de aprender. }\end{array}$ \\
\hline $\begin{array}{l}\text { Coronel } \\
(2018)\end{array}$ & $\begin{array}{l}\text { Neuroeducación emocional } \\
\text { y discapacidad intelectual }\end{array}$ & $\begin{array}{l}\text { Los niños con discapacidad } \\
\text { intelectual viven emociones más } \\
\text { intensas, por lo cual se requiere que } \\
\text { sean reguladas de una manera } \\
\text { adecuada, cuando se presentan } \\
\text { emociones positivas, se libera } \\
\text { dopamina, un neurotransmisor que } \\
\text { influye notablemente en los } \\
\text { procesos atencionales, fortalece la } \\
\text { memoria, ayuda en el análisis, la } \\
\text { toma de decisiones y la } \\
\text { planificación del futuro, también } \\
\text { favorece de manera significativa en } \\
\text { la creatividad. }\end{array}$ \\
\hline
\end{tabular}




\begin{tabular}{|c|c|c|}
\hline $\begin{array}{l}\text { Guillen } \\
\text { (2015) }\end{array}$ & $\begin{array}{l}\text { Actividades } \\
\text { neuropedagógicas basadas } \\
\text { en lúdica y recreación. }\end{array}$ & $\begin{array}{l}\text { La novedad alimenta la atención } \\
\text { sostenida }\end{array}$ \\
\hline $\begin{array}{l}\text { Vaquerizo. } \\
\text { (2019) }\end{array}$ & $\begin{array}{lrr}\begin{array}{l}\text { Procesos } \\
\text { deporte yeuronales, } \\
\text { pedagógicas. }\end{array} & \text { prácticas } \\
& \end{array}$ & $\begin{array}{l}\text { Por medio de la actividad física, se } \\
\text { fortalece la memoria a largo plazo y } \\
\text { un aprendizaje más eficiente, } \\
\text { puesto que al aportar oxígeno al } \\
\text { cerebro se optimiza su } \\
\text { funcionamiento y ocasiona una } \\
\text { liberación de noradrenalina y } \\
\text { dopamina, que son de gran } \\
\text { importancia en los diferentes } \\
\text { procesos atencionales. }\end{array}$ \\
\hline $\begin{array}{l}\text { Cohen } \\
\text { (2016) }\end{array}$ & $\begin{array}{l}\text { Interacción y participación } \\
\text { educativa a través del juego }\end{array}$ & $\begin{array}{l}\text { A través del juego, el niño con } \\
\text { discapacidad vive emociones } \\
\text { positivas que se dan por medio del } \\
\text { juego que contribuyen en el tejido } \\
\text { neuronal y en la liberación de } \\
\text { dopamina, la cual se encarga de } \\
\text { estimular la producción de } \\
\text { neuropéptidos, de esta manera, se } \\
\text { forja la plasticidad y se consolidan } \\
\text { las sinapsis, lo } \\
\text { que permitirá una maduración } \\
\text { funcional del cerebro }\end{array}$ \\
\hline
\end{tabular}




\begin{tabular}{|c|c|c|}
\hline $\begin{array}{l}\text { Rojas } \quad \text { y } \\
\text { González } \\
\text { (2017). }\end{array}$ & \begin{tabular}{|lr} 
Trabajo cooperativo y niños \\
con & discapacidad \\
intelectual &
\end{tabular} & $\begin{array}{l}\text { El trabajo cooperativo conlleva al } \\
\text { niño con discapacidad intelectual a } \\
\text { socializar con los compañeros de } \\
\text { escuela y a sentirse integrado } \\
\text { dentro de ella, lo que mejorará el } \\
\text { estado de ánimo que es } \\
\text { fundamental para un óptimo } \\
\text { aprendizaje }\end{array}$ \\
\hline $\begin{array}{l}\text { Viñamagua } \\
\text { (2018). }\end{array}$ & Pictograma & $\begin{array}{l}\text { El pictograma es muy útil para } \\
\text { facilitar la comprensión lectora }\end{array}$ \\
\hline Tilleria (2019) & $\begin{array}{l}\text { Evaluación formativa, desde } \\
\text { una perspectiva } \\
\text { neuroeducativa }\end{array}$ & $\begin{array}{l}\text { La flexibilidad en la evaluación en } \\
\text { niños con discapacidad intelectual } \\
\text { es un factor muy importante para } \\
\text { mantener su motivación en el } \\
\text { aprendizaje y no permitir que caigan } \\
\text { en la frustración al no alcanzar los } \\
\text { mismos logros que los demás } \\
\text { compañeros }\end{array}$ \\
\hline García (2014) & $\begin{array}{l}\text { Música para amenizar } \\
\text { mientras el niño con } \\
\text { discapacidad } \\
\text { aprende }\end{array}$ & $\begin{array}{l}\text { La música es una actividad } \\
\text { armoniosa, dinámica, sensorial, que } \\
\text { permite que el estudiante evite } \\
\text { distracciones al convertir la } \\
\text { actividad en algo agradable. }\end{array}$ \\
\hline $\begin{array}{l}\text { Piñeiro } \\
\text { (2021) }\end{array}$ & $\begin{array}{l}\text { Neuroaprendizaje } \\
\text { discapacidad intelectual. }\end{array}$ & $\begin{array}{l}\text { Los estudiantes con discapacidad } \\
\text { intelectual presentan menor control } \\
\text { atencional y menor capacidad de } \\
\text { memorizar, por lo cual actividades } \\
\text { que refuercen estas funciones } \\
\text { cognitivas permitirán un mejor }\end{array}$ \\
\hline
\end{tabular}




\begin{tabular}{|l|l|l|}
\hline & $\begin{array}{l}\text { proceso para adquirir la } \\
\text { información. }\end{array}$ \\
\hline
\end{tabular}

Fuente: Elaboración propia con adaptación de los autores: Maldonado (2016), Willingham (2016), Coronel (2018), Guillen (2015), Vaquerizo (2019), Cohen (2016), Rojas y González (2017), Viñamar (2018), Tilleria (2019), Garcia (2014), Piñeiro (2021).

En la tabla anterior, se puede evidenciar la postulación de diferentes estrategias neuroeducativas por diferentes autores que pueden ser provechosas para los niños con discapacidad intelectual, sin embargo, es importante tener en cuenta que no hay estrategias neuroeducativas mejores o peores, el ambiente escolar, los conocimientos ya obtenidos, la actitud del estudiante o sus condiciones de discapacidad intelectual, establecerán en cada situación la estrategia a adoptar, o su replanteamiento, según sea el caso, sin embargo a continuación se complementará de manera más detallada cada una de las estrategias:

\section{Experiencia sensorial, discapacidad intelectual y pedagogía.}

Los niños con discapacidad intelectual no pueden percibir de manera global lo que sucede en el entorno si usan un solo sentido; para que el cerebro pueda forjar conceptos de significación más completos es esencial usar los cinco sentidos. "Todas las vías de percepción sensorial son caminos de acceso al cerebro, por lo que todos los datos informativos que ingresen por ellas pueden ser procesados por él" (Soler, Citado por García, 2015, p.8). Cuando se preparan los canales de entrada sensoriales, se fortalecen habilidades cognitivas básicas que contribuirán a un aprendizaje significativo en cada área del saber, además brinda estrategias que son muy eficientes para los niños con DI, porque los convierte en personas más receptivas que sintonizan mejor con el entorno. (De la Fuente, 2015).

Las emisiones de señales externas permiten la modificación y construcción de circuitos neuronales, a través de los órganos sensoriales, para lo cual es necesario un sustrato maleable en el SNC, de esta manera se dará la neuroplasticidad y posteriormente se producirá el aprendizaje. (Martínez, 2017).

Por otra parte, todo lo que es captado por los sentidos produce un aprendizaje, "si el niño tiene una serie de conocimientos adecuados y suficientes puede desarrollar habilidades como: la observación, análisis, ordenamiento, clasificación, 
representación, memorización, interpretación y evaluación, operaciones mentales que constituyen las habilidades cognitivas básicas”. (De Sánchez, 2015, p.12).

\section{La plasticidad cerebral como componente neuroeducativo, en relación con la discapacidad intelectual.}

La experiencia modifica el cerebro de manera continua, generando las sinapsis que fortalecen el aprendizaje por medio del proceso de regeneración neuronal. (Guillen, 2015).

Para fortalecer el aprendizaje el cerebro requiere de la repetición para consolidar lo que va a asimilar, pues es a través de automatismos que se logra la memoria de trabajo. Willingham (2015).

\section{Neuroeducación emocional y discapacidad intelectual}

Los niños con discapacidad intelectual poseen emociones mucho más intensas que aquellos que no la tienen, por tanto, una de las mayores dificultades que van a presentar en cuanto al desarrollo afectivo-emocional va a ser el control sobre estas emociones, por eso la importancia de una regulación de éstas. (Coronel, 2018).

Muchos autores se han basado para la creación de sus teorías en la afirmación planteada por Platón hace más de 2000 años, la cual es "todo aprendizaje tiene una base emocional" y muchos estudios de hoy en día así lo evidencian. "Las emociones se fundamentan en una compleja red de zonas cerebrales, muchas de las cuales están también implicadas en el aprendizaje. Algunas de estas regiones del cerebro son el córtex prefrontal, el hipocampo, la amígdala o el hipotálamo". Lang, (2016, p.3). Según lo expuesto anteriormente se evidencia que ante estados de bienestar se optimiza el proceso de aprendizaje, es por ello que el docente debe buscar la forma de que el estudiante esté motivado mientras está aprendiendo.

\section{Actividades neuropedagógicas basadas en lúdica y recreación dirigidas a niños con discapacidad intelectual}

Estudios científicos han comprobado que para mantener la atención, no es importante la recompensa, sino la expectativa que ésta genera. Se ha comprobado que la liberación de dopamina se genera cuando el organismo tiene alguna expectativa. Guillen.(2015). 
Ante lo anterior, se establece que, tanto en el nivel neuronal como en la conducta, lo importante para mantener la atención, es la expectativa que genera la entrega del premio, y no la recompensa en sí.

\section{Procesos neuronales, deporte y prácticas pedagógicas.}

Al realizar actividad física el cerebro recibe más oxígeno, se forman nuevos vasos sanguíneos, e incrementan los niveles de serotonina, la cual contribuye en la memoria, Barrios (2016), así mismo, la actividad física cumple un papel muy importante en las personas con discapacidad intelectual, pues mejora la autoestima y se tiene la motivación para la superación del logro, también ejerce una influencia importante en funciones ejecutivas tales como la resolución de problemas y el control de impulsos. (Guillen, 2015).

\section{Evaluación formativa, desde una perspectiva neuroeducativa}

Otra de las estrategias para reestructurar el modelo pedagógico desde la neuroeducación es la forma cómo se evalúan los estudiantes.

Por tal razón sería relevante, entonces, que los educadores abandonaran la mezquindad de las calificaciones, el número, el $\mathrm{Cl}$, la cuantificación y pudieran situarse en las cualidades diversas de cada aprendiente, evaluando saberes y aprendizajes de manera integral, procesual y continua, sumativa y formativa, comprendiendo que niños y niñas además de aprender de manera visual, auditiva, táctil, olfativa, lingüística y lógica, también tienen la exacta capacidad de aprender de manera verbal, analítica, reflexiva, intuitiva, impulsiva, global, conceptual, perceptiva, motora, interpersonal, intrapersonal y emocional (Tilleria, 2019, p.73)

\section{Neuroaprendizaje y discapacidad intelectual.}

En la discapacidad intelectual se reconocen etapas sensibles y críticas donde se presentan dificultades en el reconocimiento y recuperación de la información. Por tal motivo, se recomiendan actividades de memoria y atención, como tareas de copia y construcción de sujetos u objetos, mediante los canales sensoriales, conservando la relación semántica, a través de acontecimientos reales o imaginarios (Piñeiro, 2021). 
Interacción y participación educativa a través del juego como estrategia de intervención en niños con discapacidad intelectual

En la discapacidad intelectual se identifican barreras pedagógicas frente al reconocimiento de habilidades lógico matemáticas, mostrando demora en la adquisición de pensamientos abstractos. Sin embargo, Aristizábal, Colorado y Gutiérrez (2016) proponen la estrategia continua, colaborativa e incluyente del juego motivacional, lúdico y participativo en el aula y en el sistema familiar. Esto se puede lograr, mediante vivencias experimentales que expongan al estudiante al desarrollo de pensamiento analógico, y a la resolución de problemas reales.

\section{Trabajo cooperativo y niños con discapacidad intelectual}

Es una técnica educativa, en la que se trabaja de manera grupal o en parejas, que permite la retroalimentación del otro a la medida que se ejecuta la tarea. (Rojas y González, 2017).

\section{Pictogramas que favorecen la comprensión en niños con discapacidad intelectual}

Estos gráficos se usan en primera instancia con niños que presentan necesidades educativas especiales, pues facilitan la comunicación al conservar el mensaje que se desea transmitir y el niño podrá comprender determinado concepto de una manera más rápida, por medio del sentido de la vista, favoreciendo de esta manera, la estructuración adecuada del lenguaje y se disminuye la ansiedad, al anticipar las actividades que se harán durante el día. (Viñamar, 2018).

\section{Música mientras el niño con discapacidad intelectual aprende}

Estudios científicos han demostrado que las actividades relacionadas con artes, en particular la música, promueven el desarrollo de procesos cognitivos, como efecto de la plasticidad cerebral. Posner.(2015).

Según Changeux y Manouri (2016), la música activa el sistema límbico de las emociones, lo que también implica un fortalecimiento en el aprendizaje.

Adicional a lo anterior es muy importante tener en cuenta que la poda en el sistema nervioso se genera por las experiencia, cuando en la infancia no se tienen estímulos adecuados, se ocasiona una pérdida de la poda, lo que implica que hay una pérdida de capacidades con el tiempo, por ello, el desafío de una educación basada en la plasticidad cerebral. Pascual. (2015). 
Cuando el estudiante es el protagonista activo del aprendizaje, es allí, donde éste se optimiza y se facilita cuando es una actividad placentera, el cerebro permite mejorar y el ser creativo, es por ello que la neuroeducación resulta fundamental en aquellos estudiantes con discapacidad intelectual Guillen. (2015).

A continuación se presenta el análisis e interpretación de investigaciones sobre las estrategias de neuroeducación aplicadas a la discapacidad intelectual, en el aula. Estos trabajos de carácter descriptivo y experimental permiten obtener interesantes conclusiones, donde se puede constatar el impacto positivo que tienen las técnicas de Neuroeducación en el mejoramiento del rendimiento escolar.

La Fundación Saldarriaga Concha (2015) presenta un informe alternativo sobre los avances de la participación e inclusión de las personas en condición de discapacidad intelectual en Colombia, reconociendo que de 10.3 millones de estudiantes matriculados en el sistema educativo colombiano el $37.9 \%$ logra finalizar primaria, el $20.7 \%$ completa con complejidad y obstáculos permanentes la secundaria; el $1.7 \%$ es guiado por redes de apoyo constantes para finalizar la educación superior y el $33.8 \%$ no inicia ningún proceso formal de aprendizaje (Correa y Castro, 2016). Evidenciando así, un alto índice de deserción escolar a medida que se complejizan los procesos de enseñanza y la extensión de barreras actitudinales, sociales y físicas que construyen desde el sistema familiar y se extienden hasta el contexto social y cultural del niño (Ranz y Gimenéz, 2018).

A causa de lo encontrado anteriormente por la Fundación Saldarriaga Concha en el (2015), las instituciones educativas deben reconocer la influencia de las creencias, percepciones y opiniones de los padres y educadores para generar cambios significativos en la reestructuración de currículos flexibles (Ke, Liu 2017); reconociendo que la ausencia y abandono de niños en condición de Dl en el aula, puede ser generado por desmotivación de no saber construir y responder un ambiente de enseñanza,juego,aprendizaje y colaboración entre disciplinas que trabajan de forma desarticulada, en base a constructos biológicos, principios pedagógicos y modelos multidimensionales (Miller, 2016).

Por tal razón, Puentes y Sanchez (2019) proponen un investigación empírica y teórica, sobre la formación profesional de docentes guiados por la neurociencia en base a prácticas incluyentes; reconociendo necesidades significativas en la formación profesional de los educadores para generar un impacto significativo en la educación y evaluación formativa de la DI Tilleria (2019); siendo así que de una muestra premeditada de 14 profesores de la Universidad de Pinar del Río, en Cuba; el $100 \%$ de los participantes poseen estudios en master en educación, el $24,4 \%$ 
son doctores en ciencias pedagógicas y el $35,75 \%$ de participantes han tenido más de 10 años de experiencia en logopedia. Los resultados arrojaron que los currículos diseñados en la licenciatura de Educación especial y en logopedia cuentan con la incorporación de contenidos pseudocientíficos que son insuficientes y poco prácticos en la articulación de las competencias propuestas desde la pedagogía. Donde se exponen de forma independiente cada temática, sin enlazar la funcionalidad y el objetivo de trabajar desde las redes neuronales y las estrategias neuroeducativas. (Alagarda y Giménez 2018).

Para validar la concepción sobre la importancia de la investigación propuesta por Alargada y Giménez en el 2018, se presenta un trabajo de investigación de la Universidad de los Andes de Venezuela con alumnos cuyas edades variaron entre 17 y 21 años; donde se evalúa la influencia de la neuroeducación en el rendimiento académico en una asignatura determinada (Ferrer, Morelo, Leal, Añez, Araque y Ávila, 2020), identificando que los ambientes enriquecidos con prácticas basadas en ciencias del cerebro, evidenciaban resultados académicos positivos en el desarrollo de competencias de ser y saber hacer (Gómez, Muriel y Londoño, 2019).

El Índice de aprobación en la asignatura de los grupos experimentales mediante prácticas neuroeducativas fue del 90,6\%, contra el $47 \%$ del grupo control que maneja estrategias tradicionales de educación; reforzando la afirmación de cómo la relación entre la pedagogía y la neuroeducación puede cambiar el rol pasivo del estudiante y la familia en aprendices y constructores activos del aprendizaje colaborativo y personal ( Codine, 2015). Figueroa y Farnum (2020) destacan que, la apertura de aprendizajes novedosos y motivacionales generan cambios significativos en el aula, y la necesidad del estudiante por aprender, divirtiéndose.

En concordancia con lo anterior, es importante reconocer que"un ambiente enriquecido y psicológicamente adecuado posibilita el crecimiento de nuevas neuronas, especialmente en el hipocampo, mejorando el aprendizaje, de manera que las situaciones divertidas y emocionalmente positivas, potencian el aprendizaje y fijan rápidamente los contenidos curriculares y el interés en el tema de clase". Ferrer et al (2020).

De forma similar, se presenta un estudio construido en Valladolid España, donde se plantea el objetivo de investigar el proceso atencional de la atención y la ansiedad de los alumnos en el aula, y el impacto de las estrategias de neuroeducación en la evaluación e intervención de estos síntomas encontrando que, después de la intervención neuroeducativa en el aula, la atención tenía un crecimiento del $20 \%$ y en consecuencia, el cumplimiento de las normas de clase 
había aumentado considerablemente, los estudiantes mostraban una disminución en movimientos corporales, demostrando que esta reducción generaba mayor control de impulsos.en relación con las cifras obtenidas antes de la intervención. Al verse reducida la ansiedad y el nivel de atención aumentado, se había conseguido disminuir las conductas disruptivas. El impacto permitió pasar de un $33,34 \%$ de conductas inadecuadas previo a la intervención, a solo el $6,67 \%$ luego de la intervención Neuroeducativa en el aula. (Bategon, 2020).

Los trabajos investigativos planteados con anterioridad son respaldadas por Martínez, Piqueras, Delgado y García, (2018) dichos autores postulan una revisión detallada de artículos cualitativos y cuantitativos sobre las contribuciones de la neuroeducación a las competencias y adaptaciones curriculares,concluyendo que los beneficios que envuelve la implementación del conocimiento cerebral en la construcción de estrategias educativas, en la resolución de problemas y en el control emocional desencadenan procesos de motivación, iniciativa y aprendizaje para las demandas evolutivas según edad y necesidad educativa. Howard et al. (2016).

Esto demuestra que gracias a las prácticas neuroeducativas en el aula pueden generarse cambios significativos en determinados aspectos evaluados, como lo fueron en este caso la atención y la ansiedad en la clase.

\section{Estrategia metodológica}

Para poder desarrollar este trabajo de investigación se realizó un exploración de 30 artículos de revistas indexadas que guardan una estrecha relación con discapacidad intelectual, siguiendo el lineamiento de "Resumen Analítico especializado de artículos científicos" (RAE), en el cual 15 artículos pertenecen a discapacidad intelectual y 15 artículos pertenecen a neuroeducación con una ventana de observación de 5 años, desde el año 2015 a 2020, en su mayoría se utilizó investigación cualitativa de carácter descriptivo y exploratorio, algunos artículos son colombianos, por lo cual se acerca a la población de estudio del presente trabajo y algunos artículos son de otros países en vía de desarrollo, que de alguna forma evidencian una diferencia significativa en cuanto a cifras de niños con discapacidad intelectual, por lo cual en países como Colombia surge una necesidad inminente de brindar la intervención adecuada. 
Por consiguiente, muchos autores coinciden en que la neuroeducación es una herramienta fundamental en el aprendizaje de niños con discapacidad intelectual.

Para llevar a cabo este estudio, se realizó una investigación con enfoque cualitativo en la que se obtuvo información por medio de hallazgos de diferentes autores que permiten la comprensión de las diferentes problemáticas frente a la discapacidad intelectual y se determinó la influencia que ejerce la neuroeducación mediante un estudio de tipo exploratorio, siendo el propósito fundamental examinar un tema o problema de investigación poco estudiado, del cual se tienen muchas dudas. (Hernández, Fernández y Baptista, 2003), es importante señalar que este tema es novedoso y se considera que es importante que los docentes lo tengan en cuenta en sus aulas puesto que contribuye a la solución para esta población escolar.

\section{Diseño}

Es de carácter no experimental, de tipo transeccional-descriptivo puesto que, en este estudio, el interés radica en describir una problemática y los beneficios de las estrategias a utilizar mediante el análisis y presentación de los datos recopilados sin acudir a la manipulación de alguna variable.

\section{Estrategia de análisis}

La revisión bibliográfica es el paso previo que se da antes de comenzar cualquier proceso investigativo. Esta actividad fundamental permite conocer a profundidad el tema e identificar los trabajos ya existentes acerca de las diferentes perspectivas con los cuales se ha trabajado. Esta cuidadosa revisión de la literatura permite ponerse al día sobre los avances e innovaciones académicas que actualmente se llevan a cabo.

\section{Discusión}

El objetivo de la presente investigación es mostrar cómo la neuroeducación favorece el desarrollo integral de niños en condición de discapacidad intelectual en el aula; frente a esto, es preciso reconocer que las investigaciones citadas concluyen en sus resultados que, la práctica pedagógica necesita ser transformada para poder vincular el proceso de enseñanza y aprendizaje con el funcionamiento cerebral. Figueroa \& Farnum (2020). En relación con los resultados presentados, se encontró que la neuroeducación es concebida como una interdisciplina que se construye a partir de conocimientos e investigaciones científicas. Pallarés (2016). 
Que se redefinen, evalúan y se transforman por medio de teorías, conocimientos, principios y procedimientos pedagógicos que tienen como objetivo convertir los desafíos neuronales en oportunidades de aprendizaje incluyentes y participativas para los niños y niñas en condición de discapacidad intelectual. Flórez (2015).

Al conceptualizar la discapacidad intelectual desde la mirada de la neuroeducación; y su incidencia en la calidad de vida de los niños y niñas en el aula, se demostró que, la comunidad educativa carece de conocimiento para evaluar, intervenir, socializar y formar currículos, entornos y aulas basadas en aprendizajes diversos e incluyentes. Alargada y Giménez. (2018). Debido a barreras de acceso actitudinales y pedagógicas que se desarrollan desde el cumplimiento de parámetros y estereotipos sociales, que muestran la condición de discapacidad intelectual como una invalidez neurológica, emocional y psicosocial que limita al estudiante hacer un sujeto pasivo dentro del aula. Caballero (2018).

Un estudio realizado por Martínez, Piqueras, Delgado y García (2018), explica cómo los saberes neuronales en la práctica educativa pueden facilitar al educador el desarrollo de tareas y objetivos pedagógicos, puesto que se entrena para enseñar al estudiante aprender desde la motivación, resolución de problemas y el trabajo cooperativo. Es ahí donde la neuroeducación tiene el objetivo de aportar al sistema familiar y a la comunidad educativa, estrategias para comprender los mecanismo de aprendizaje y el desenvolvimiento socio afectivo, en base al proceso cerebral ( Santana, 2019).Desde el punto de vista de Codina (2015), en su estudio sobre la neuroeducación, se expone la necesidad de la comunidad educativa por comprender los procesos cognitivos y su relación con las alteraciones de aprendizaje y conducta, dando a conocer así que la neurociencia y la pedagogía se buscan continuamente para consolidar una disciplina que supla en totalidad las necesidades en relación con y sin dificultades de aprendizaje; y su incidencia en el comportamiento en el aula. Verdugo, Córdoba y Rodríguez (2020).

Por consiguiente, dentro del ambiente educativo crece la esperanza de facilitar oportunidades de cambio para las personas en condición de discapacidad intelectual, mediante el reconocimiento de agentes biológicos encargados de la organización y asimilación de patrones de comportamiento y aprendizaje; los cuales son liderados por sistemas neuronales que se fortalecen según el grado de experiencias y vivencias socio-afectivas. Corona, Peñaloza y Vargas. (2017).

Este argumento es reforzado por la revisión bibliográfica realizada por Medel y Camacho (2019) reconociendo que, los tecnicismos científicos no se deben establecer como una barrera de acceso, sino, como una oportunidad de 
acercamiento hacia la inclusión educativa, donde el objetivo sea integrar, entrenar, educar y acompañar a los docentes en la comprensión de los términos y metodología que se desarrolla en las investigaciones, por parte de los profesionales de la salud. Sin embargo, se logra reconocer en el estudio realizado por Puentes y Sanchez (2019) que, desde el proceso de formación de profesionales de la educación existe y se mantiene una necesidad de conexión, comunicación y vinculación constante de conocimientos entre el desarrollo cerebral y los procesos de aprendizaje.

Dentro de los hallazgos encontrados, se identificaron varias estrategias neuroeducativas favorecedoras de los procesos de desarrollo y educación para los niños y niñas en condición de discapacidad; aprendizajes basados en variables sensoriales, cognitivas, neuropedagógicas y socioemocionales, que permitieron reconstruir la relación entre la educación y las neurociencias; y su impacto en la práctica educativa Béjar (2015). En primer lugar, se encontraron investigaciones y aportes experienciales, a través de canales sensoriales y la plasticidad neuronal. Willingham (2015), que resumen la enseñanza desde vivencias, experimentación y la novedad en la construcción de prácticas autodidactas, para luego formar conocimientos colaborativos Guillen (2015). En complemento, un estudio realizado por Maldonado (2016), sobre la eficacia de la estimulación sensorial en niños con un grado de discapacidad intelectual, arrojó que la exploración del mundo a través de los sentidos contribuye al ritmo de aprendizaje de los estudiantes de forma lúdica y pedagógica.

En contraste con la información anterior, aparecen las contribuciones expuestas por los autores Araya y Espinoza (2020); sobre cómo desde los procesos neuronales se contribuye de manera positiva las emociones, la empatía y la consolidación de conocimientos, habilidades y destrezas cognitivas y psicoafectivas; registrando y asimilando la información desde áreas cerebrales encargadas de la discriminación y asociación emocional, como el hipocampo y la amígdala (Coronel, 2018). Este mismo planteamiento había sido corroborado por Aristizábal, Colorado y Gutiérrez (2016), en su investigación experimental sobre el juego, con estudiantes de grado quinto de primaria, reconociendo las actividades lúdicas como instrumentos para motivar el aprendizaje en el aula, facilitando metodologías que ponen en práctica las habilidades de interacción social, planeación y organización de estrategias, resolución de conflictos y el pensamiento analógico. Rojas y González (2017). De igual importancia, Garcia y Rubio (2020), en su estudio sobre el impacto de la música en la calidad de vida de las personas en condición de discapacidad en el aula, afirman que los estudiantes durante los espacios en los que se incluye la música fortalecen o desarrollan conductas adaptativas y emociones positivas que 
promueven contextos de participación igualitaria con sus pares y figuras de autoridad.

Por otro lado, Tilleria (2019) propone una resignificación de los métodos evaluativos, en la construcción de aulas neuroeducativas, donde debe ser resaltada la variedad y el respeto por los procesos formativos, registrando los logros, avances y dificultades de los estudiantes en condición de discapacidad intelectual, para diagnosticar y diseñar un plan único de flexibilización e inclusión. Para ejemplificar el postulado anterior, Estupiñán y Valverde (2021) proponen la pertinencia que los docentes autoevalúan la efectividad de qué, cómo y para qué enseñan, con el fin de construir aulas accesibles.

En consecuencia con los planteamientos anteriores, se logró describir por medio de un estudio sobre la deserción escolar en estudiantes con discapacidad de Fuentes en el (2013) que, las propuestas implementadas para reestructurar y flexibilizar las prácticas educativas y sociales de los estudiantes en condición de discapacidad en Colombia, se encuentran en un proceso de ajuste y adaptación a la normalización e individualización de las necesidades únicas de cada estudiante en condición de discapacidad (MEN, 2015). Puesto que, los procedimientos institucionales y socioemocionales se muestran carentes de estrategias para abordar las temáticas y los contenidos por parte de los docentes en el proceso de aprendizaje y enseñanza con los estudiantes y el sistema familiar. Cabrera, Lizarazo \& Medina (2016). Por otra parte, se propone la ley 1346 de 2009, aprobada por la Corte Constitucional Colombiana, la cual promueve los derechos de los niños y niñas en condición de discapacidad desde la inclusión educativa, bajo las mismas condiciones de sus pares, promoviendo oportunidades para alcanzar la equidad. Araya y Espinoza ( 2020).

Como consecuencia, Brugué (2015) en su investigación sobre " ¿Discapacidad o diversidad funcional?" afirma que la sociedad ha construido las deficiencias en base a la discapacidad; limitando, restringiendo y discriminando al individuo con discapacidad intelectual por su impresión diagnóstica; olvidando la importancia de involucrar el desarrollo personal que puede transformar las limitaciones, en habilidades para la vida (Ortiz, 2018). Del mismo modo, la Fundación Saldarriaga Concha en el ( 2015) ,presenta que más del 60\% de estudiantes con discapacidad en Colombia mantiene un proceso educativo desamparado de derechos y recursos para suplir las necesidades que conlleva una condición de vida contraria a lo planificado desde la enseñanza tradicional. Cabrera, Lizarazo \& Medina (2016). Por lo que, el estigma de "discapacidad" será restituido y transformado con la principal fuente de apoyo y protección, la familia. Contreras (2015), son ellos, los 
encargados de instaurar y promover espacios de entrenamiento, estimulación y colaboración; identificando las capacidades que los niños en condición de discapacidad aún no poseen, pero que con evaluación e intervención pedagógica y terapéutica pueden desarrollar de forma autónoma. Almario (2016).

Para finalizar, se propone plantear, desde lo que menciona Brugué (2015), cambiar el término de discapacidad intelectual por "diversidad funcional", reconociendo que no se posee una discapacidad, sino se es diverso desde su apreciación; dejando de lado la marginación y el rechazo de la sociedad por la diferencia.

\section{Conclusiones}

A partir de la revisión bibliográfica de diferentes autores sobre neuroeducación y la actividad de investigación desarrollada, se puede concluir que la neuroeducación favorece el desarrollo integral de los niños en condición de discapacidad intelectual en el aula. Se entiende la Neuroeducación como una disciplina que construye su conocimiento, a partir de investigaciones científicas y que sus resultados deben aportar, tanto a la familia como a la comunidad educativa, estrategias para comprender los mecanismos de aprendizaje y desenvolvimiento socio afectivo en base al proceso cerebral.

Los educadores y las instituciones educativas tienen una gran responsabilidad, la cual consiste en incluir, a niños con discapacidad intelectual, y que ellos alcancen un aprendizaje integral que les permita una mejora en la calidad de vida, tanto suya como la de sus familias. El poner en práctica estas estrategias les generará un valor mayor a estas instituciones que simplemente ser reconocidas como instituciones educativas incluyentes.

Para ello se requiere una preparación ardua del equipo docente, ya que su misión no será solo la de brindar conocimientos, también deben comprender cómo aprende y funciona el cerebro de un niño con discapacidad intelectual para poder brindar las estrategias adecuadas. Medel y Camacho (2019), en sus trabajos de investigación establecen que los tecnicismos científicos no deben ser una barrera de acceso sino una oportunidad de acercamiento hacia la inclusión educativa con el objetivo de integrar, entrenar, educar y acompañar a los docentes. Más aún Puentes y Sánchez (2019) afirman que, desde el proceso de formación de los profesionales de la educación, estos deben tener acceso al conocimiento de la relación que existe entre el desarrollo cerebral y los procesos de aprendizaje.

Sin el uso de estas estrategias, solo utilizando la pedagogía tradicional, el trabajo del aula se puede volver frustrante, tanto para el docente como para el estudiante, 
afectando de esta manera la autoestima del niño cuando percibe que no puede aprender lo que se le está enseñando y en el peor de los casos, se puede perjudicar su desarrollo neurológico. Es fundamental optimizar de manera adecuada el tiempo en pro de la intervención adecuada que requiere el niño elaborando un adecuado diagnóstico de su discapacidad intelectual. La manera en que se aproveche el tiempo en los niños con esta condición es muy importante en su desarrollo, no se debe tomar a la ligera el proceso de enseñanza de un niño que presente discapacidad intelectual, o cualquier trastorno del aprendizaje.

Por otra parte, cabe resaltar el rol fundamental que tienen las emociones en el aprendizaje, especialmente en el caso de niños diagnosticados con discapacidad intelectual que presentan emociones más intensas, y muchas veces padecen trastornos de ansiedad, entre otros. Trejar. (2016). Esto implica conocer los mecanismos para regular las emociones que impiden un adecuado aprendizaje y tener en cuenta las estrategias mencionadas anteriormente. Cuando el alumno no se encuentra motivado, sea cual sea su condición, así se le presente una clase magistral, bien preparada, lograr captar la atención del estudiante será una misión casi imposible.

En ese sentido, lo anterior invita al desarrollo de actividades de investigación y prácticas rigurosas en el marco de la neuroeducación en el contexto de las instituciones educativas, como se mencionaba anteriormente. Existen numerosos factores, tanto emocionales como de diverso tipo, los cuales afectan e inciden en los procesos de aprendizaje y plantean nuevas preguntas que requieren adecuadas respuestas, con el fin de mejorar los métodos de aprendizaje, en los niños con alguna limitación en su aprendizaje.

En el caso del presente trabajo, el acceso y énfasis que se realizó en el estudio y análisis de numerosos y variados trabajos de investigación realizados en diferentes contextos ha permitido que se incrementen los elementos de juicio en el estudio de las neurociencias y apreciar en mayor medida su relación con los procesos de aprendizaje. Este aspecto se considera como una muy útil contribución para la formación como estudiantes en neuropsicología escolar.

Un último aspecto para señalar consiste en la necesidad de reflexionar sobre los cambios importantes que estos conocimientos generan en la práctica educativa, en el salón de clase. El dinamismo que ellos conllevan implica una revisión constante de las estrategias de educación que permitan subsanar los vacíos, que, en los 
procesos de educación tradicionales, se mantienen por la ausencia de programas de capacitación en los docentes de las instituciones educativas. Estas problemáticas, muchas correspondientes a situaciones específicas, deben ser elementos indispensables de los procesos de investigación que lleven a cabo los estudiosos de las neurociencias, para su adecuación a la práctica escolar y al logro de mejores resultados.

Sería valioso desarrollar investigaciones sobre canales sensoriales y plasticidad neuronal que resuman la enseñanza desde vivencias, experimentación y novedad. La utilización de juegos, actividades lúdicas, como instrumentos para motivar el aprendizaje en el aula se ubican en esta perspectiva. Se considera que lo anterior, facilita el desarrollo de metodologías que ponen en práctica habilidades de interacción social, planeación, organización de estrategias, resolución de conflictos, y pensamiento analógico. 


\section{Referencias}

Aguilar Aguiar, Giselvis, Demothenes Sterling, Yaima, \& Campos Valdés, Imilla. (2020). La participación familiar en la inclusión socioeducativa de los educandos con necesidades educativas especiales. Mendive. Revista de Educación, 18(1), 120-133. Epub 02 de marzo de 2020. Recuperado en 27 de agosto de 2021, de http://scielo.sld.cu/sciel o.php?script=sci_arttext\&pid=S1815-76962020000100120\&lng=es\&tIng=es.

Alargada, D. R., \& Beut, J. A. G. (2019). Principios educativos y neuroeducación: una fundamentación desde la ciencia. Edetania: estudios y propuestas socio-educativas, (55), 155-180.

Alvarado Mamani, N. E. (2016). Centro de rehabilitación para el desarrollo intelectual y físico motor de menores con discapacidad intelectual en la ciudad de Tacna.

Almario Ordoñez, E. (2016). La investigación en familias de personas con discapacidad. Paideia Surcolombiana, 21 (21), 12-26. https://doi.org/10.25054/01240307.1443

American Asociation on Intellectual and Developmental Disability (2021). Recuperado de: https://www.aaidd.org/intellectual-disability/definition.

American Psychiatric Association - APA. (2014). Manual diagnóstico y estadístico de los trastornos mentales DSM-5 (5a. ed. --.). Madrid: Editorial Médica Panamericana.

Araya-Pizarro, S.C., \& Espinoza Pastén, L. (2020). Aportes desde las neurociencias para la comprensión de los procesos de aprendizaje en los contextos educativos. Propósitos y Representaciones, 8(1). doi:http://dx.doi.org

/10.20511/pyr2020.v8n1.312

Arellano, A., y Peralta, F. (2013). Calidad de vida y autodeterminación en personas con discapacidad. Valoración de los padres. Revista Iberoamericana, 145-160 
Aristizábal Z., Jorge Hernán, \& Colorado T., Humberto, \& Gutiérrez Z, Heiller (2016). El juego como una estrategia didáctica para desarrollar el pensamiento numérico en las cuatro operaciones básicas. Sophia, 12(1),117-125.[fecha de Consulta 28 de Junio de 2021]. ISSN: 1794-8932. Disponible en: https://www.redalyc.org/articulo.oa?id=413744648009.

Barrios-Tao, Hernando (2016). Neurociencias, educación y entorno sociocultural. Educación y Educadores, 19 (3), 395-415. [Fecha de Consulta 12 de Agosto de 2021]. ISSN: 0123-1294. Disponible en: https://www.redalyc.org/articulo.oa?id=83448566005

Béjar, M. (2014). Una mirada sobre la Educación, Neuroeducación. Padres y Maestros, (355), 49-52.

Blakemore, S. J. (2016). The development of social cognition in adolescence: An integrated perspective. Neuroscience \& Biobehavioral Reviews, 70, 106-120.

Betegon Blanca,H.(2017). Neuroeducación, ansiedad y atención en alumnos de educación primaria. Tesis de maestria en Investigacion aplicada a la educación Universidad de

Bodoque-Osma, A. R. (Coord.) y González-Víllora, S. (Coord.). (2021). Neuroeducación: ayudando a aprender desde las evidencias científicas. Ediciones Morata, S. L. https://elibro.net/es/ereader/poligran/184215?page=24

BRUGUÉ, J. C. (2015). ¿Discapacidad o diversidad funcional? Siglo cero, 46(2).

Caballero, M. (2018). Neuroeducación de profesores y para profesores: de profesor a maestro de cabecera. Difusora Larousse - $\quad$ Ediciones Pirámide. https://elibro.net/es/ereader/poligran/123091?page=35.

Cabrera-García, V. E., Lizarazo-Sandoval, F.A. y Medina-Casallas, D. C. (2016). Necesidades de relaciones sociales de niños y niñas con discapacidad intelectual en la familia y en la escuela. Revista educación y desarrollo social. 10(2), 86-101. DOI: http://dx.doi.org/10.18359/reds.1554. 
Changeux, J. y Manoury, P. (2016). Las neuronas encantadas. El cerebro y la música. Barcelona, España: Editorial Gedisa

Campos, A. (2010). Neuroeducación: Uniendo las neurociencias y la educación en la búsqueda del desarrollo humano. Revista digital La Educación. OEA.

Campos, A. L. (2014). La Neuroeducación: descartando neuromitos y construyendo principios sólidos. Recuperado de http://cerebrum. la/congresomundial/papers/contenido/Anna\% 20Lucia/Neuroeducacion-ALC. pdf el, 27.

Codina Felip, M. J. (2015). Neuroeducación en virtudes cordiales: cómo reconciliar lo que decimos con lo que hacemos. Ediciones Octaedro, S.L. https://elibro.net/es/ereader/poligran/61996?page =15-22

Cohen, A. O., Dellarco, D. V., Breiner, K., Helion, C., Heller, A. S., Rahdar, A., Pedersen, G., Chein, J., Dyke, J. P., Galvan, A. \& Casey, B. J. (2016). The Impact of Emotional States on Cognitive Control Circuitry and Function. Journal of Cognitive Neuroscience. Vol. 28, Issue 3, 446-459.

Contreras Fernández, V. (2015). Discapacidad Intelectual, una oportunidad de crecimiento familiar. Revista Iberoamericana De Educación, 69(3), 157-172. https://doi.org/10.35362/rie693117

Corona Gaona, F., Peñaloza Pineda, I., \& Vargas Garduño, M. (2017). La inclusión de niños con discapacidad intelectual y en situación de calle: Una mirada comparativa entre Chile, Colombia y México. Revista Ensayos Pedagógicos, 12(2), 195-215. https://doi.org/10.15359/rep.12-2.9

Coronel, Claudia Paola. (2018). Problemas emocionales y de comportamiento en niños con discapacidad intelectual. Diversitas: Perspectivas en Psicología, 14(2), 351-362. https://doi.org/10.15332/s1794-9998.2018.0002.11

Correa Montoya, Lucas y Castro Martínez, Marta Catalina. (2016). Discapacidad e inclusión social en Colombia. Informe alternativo de la Fundación Saldarriaga Concha al Comité de Naciones Unidas sobre los derechos de las personas con discapacidad. Editorial Fundación Saldarriaga Concha. Bogotá D.C., Colombia. 162p. 
De la Fuente, R. y Álvarez, L. (2015). Biología de la Mente, México: Fondo de Cultura Económica.

De Sánchez, M. (2015). Curso: Factores del Desarrollo Intelectual. Monterrey, N.L: ITESM.

Diaz Rincon.C(2017) La odisea que tienen que vivir las personas con discapacidad en Colombia. Directo Bogotá. Recuperado de:https://www.directobogota.com

/post/2017/09/25/la-odisea-que-tienen-que-vivir-las-personas-con-discapacidad-intelectual-en-c olombia.

Estupiñan, F., Valverde, O. (2021). Estudios en la reflexión de las prácticas pedagógicas en neuroeducación. I+D Revista de Investigaciones, 16(2), 140-150.

Estupiñán, F., \& Riascos, O. V. (2021). Estudios en la reflexión de las prácticas pedagógicas y su relación con la neuroeducación. I+ D REVISTA DE INVESTIGACIONES, 16(2), 140-150.

Ferrer, K.Molero,L.Leal,A.Anez,O.Araque,M.Avila,A(2020). Influencia de la Neuroeducación en el rendimiento académico de estudiantes universitarios del área Química. Recuperado de https://www.redalyc.org/journal/356/35663284004/html/

Figueroa, Claudia, \& Farnum, Francisco. (2020). La neuroeducación como aporte a las dificultades del aprendizaje en la población infantil. Una mirada desde la psicopedagogía en Colombia. Revista Universidad y Sociedad, 12(5), 17-26. Epub 02 de octubre de 2020. Recuperado en 10 de julio de 2021, de http://scielo.sld.cu/scielo.php?script=sci arttext\&pid=S2218-36202020000500017\&lng=es\&tln $\mathrm{g}=\mathrm{es}$.

Flórez, José. (2015). Discapacidad intelectual y Neurociencia. SíNDROME DE DOWN, VOLUMEN 32, (pp 2-14).

Fuentes Ayosa, L. (2013). La deserción escolar de los estudiantes con discapacidad de las instituciones de educación especial del cantón Esmeraldas período de estudio 2011-2012 (Master's thesis).

Gallegos, J. L., \& Rodríguez, A. (2013). Bases teóricas y de investigación en educación especial. Madrid: Ediciones Pirámide. 
García García, B. ., \& Rubio Belmonte, C. (2020). Impacto de la musicoterapia en la calidad de vida de personas con discapacidad intelectual: una revisión de la literatura. Revista De Investigación En Musicoterapia, 3, 34-53. https://doi.org/10.15366/rim2019.3.003

García Alarcón, G. A. (2002). Estrategias didácticas para un aprendizaje significativo en preescolares, tesis de maestría, Toluca, México.

Gómez, L., Schalock, R., \& Verdugo, M. (2021). A NEW PARADIGM IN THE FIELD OF INTELLECTUAL AND DEVELOPMENTAL DISABILITIES: CHARACTERISTICS AND EVALUATION. Psicothema, (núm. 1, 28-35 doi: 10.7334), (PP 2-9). Retrieved from http://www.psicothema.com/english/psicothema.asp?id=4644

Gómez Vahos, Luz Estela, \& Muriel Muñoz, Luz Enid, \& Londoño-Vásquez, David Alberto (2019). El papel del docente para el logro de un aprendizaje significativo apoyado en las TIC. Encuentros, 17(02),118-131. [fecha de Consulta 8 de junio de 2021]. ISSN: 1692-5858. Disponible en: https://www.redalyc.org/articulo.oa?id=476661510011

Gross-Tur, R. ., Martinez-Rosales, Y. ., Deroncele-Acosta, A. ., \& Miller-Contreras, M. E. (2021). Gestión de potencialidades formativas de escolares con discapacidad intelectual: pautas metodológicas desde la psicología positiva. Santiago, (155), 33-48. Recuperado a partir de https://santiago.uo.edu.cu/index.php/stgo/article/view/5321

Guillén, J. (2015). Obtenido de Neuroeducación: estrategias basadas en el funcionamiento del cerebro: https://escuelaconcerebro.wordpress.com

/2012/12/27/neuroeducación-estrategias-basadas-en-el-funcionamiento-del-cerebro/

Hernández, R., Fernández, C. y Baptista, P. (2014). Metodología de la investigación. México: McGraw-Hill. 
Howard-Jones, P. A., Varma, S., Ansari, D., Butterworth, B., De Smedt, B., Goswami, U., Laurillard, D., \& Thomas, M. S. C. (2016). The principles and practices of educational neuroscience: Comment on Bowers (2016). Psychological Review, 123(5), 620-627. https://doi.org/10.1037/rev0000036

Hurtado, L. D. y A. Agudelo. (2014). Inclusión educativa de las personas con discapacidad en Colombia, Revista ces Movimiento y Salud, ISSN: 2357-562X (en línea; http://revistas.ces.edu.co/index.php/movimientoysalud/article/view/2971.

Ke, X., \& Liu, J. (2017). Discapacidad intelectual. Traducción de IRARRÁZAVAL, M., MARTIN, A., PRIETO-TAGLE, F. y FUERTES, O.). En REY, Joseph. Manual de Salud Mental Infantil y Adolescente de la IACAPAP, 1-28.

Lang, P.J. \& Davis, M. (2006). Emotion, Motivation and the Brain: Reflex Foundations in Animal and Human Research. Progress in Brain Research, Vol. 156

Maldonado Escobar, D. C. (2016). La Estimulación Multisensorial para el desarrollo Cognitivo de los Niños con Parálisis Cerebral de 2 a 3 años en la Unidad Especializada

Manjarrés-Carrizalez, D., \& Hederich-Martínez, C. (2020). Permanencias y transformaciones de los estilos parentales en la crianza de personas con discapacidad. CES Psicología, 13(2), 61-84.

Martínez-González, A. E., Piqueras, J. A., Delgado, B., \& García-Fernández, L. M. (2018). Neuroeducación: aportaciones de la neurociencia a las competencias curriculares. Publicaciones,48(2),23-34.doi:10.30827/publicaciones.v48i2.8331

Maynooth. Recuperado de http://eprints.maynoothuniversity.ie/2572/1/Tahir2011

_Thesis-Final.pdf.

Medel, M., \& Camacho, J. (Julio de 2019). La neurociencia aplicada en el ámbito educativo. el estudio de los mitos. International Journal of New Education(3), 70-81.

Mercurio, E. (2016). Pobreza y discapacidad intelectual en el sistema penal: los invisibles. Revista $\begin{array}{lllll}\text { Argentina de Psiquiatría, 27, } & 197-207 . & \text { Recuperado de } \\ \text { http://www.pensamientopenal.com.ar/system/files/2016/09/doctrina44166.pdf } & \end{array}$

Ministerio de Educación (2015). Recuperado de_\}: http://www.mineducacion.gov.co 
Ministerio de Salud y Protección social de Colombia (2018) Recuperado de https://www.minsalud.gov.co/proteccionsocial/promocionsocial/Discapacidad/Paginas/registr o-localizacion.aspx

Mora, F. (2017). Neuroeducación: solo se puede aprender aquello que se ama. Difusora Larousse - Alianza Editorial.https://elibro.net/es/ereader/poligran/122878?page=21-28

Montero, M. M., \& Conde, J. A. C. (2019). La neurociencia aplicada en el ámbito educativo. El estudio de los neuromitos. International Journal of New Education, 2(1

Organización Mundial de la Salud (2016), Recuperado de: https://www.who.int/es.

Ortiz-Quiroga, M., Ariza, Y., \& Pachajoa, H. (2018). Calidad de vida de familias de niños y adolescentes con discapacidad asociada a defectos congénitos. Universitas Psychologica, 17(1), 1-10. https://doi.org/10.11144/Javeriana.upsy17-1.c vfn.

Pallarés-Domínguez, D. (2016). Neuroeducación en diálogo: neuromitos en el proceso de enseñanza-aprendizaje y en la educación moral

Pascual, A. Promoviendo la Salud Cerebral. Conferencia ofrecida en el marco de la celebración del 50 aniversario del Institut Guttmann. 5 de octubre de 2015. Barcelona.

Piñeiro, B. (2021). NEUROEDUCACIÓN. Emociones y aprendizaje [Libro electrónico]. En NEUROEDUCACIÓN. Emociones y aprendizaje (9789582013509.a ed., pp. 94-99). Magisterio.

Posner, M. et al. (2015). Arts education, the brain, and language. In C. et. al. Asbury (Ed.), Learning, Arts, and the Brain: The Dana Consortium Report on Arts and Cognition,

Presidencia de la República de Colombia. (Agosto 29, 2017). Decreto 1421 de 2017. Decreto de Ministerio de Educación Nacional,https://www.http://es.presidencia.gov.co/normativa/normativa/DECRETO\%201421\% 20DEL\%2029\%20DE\%20AGOSTO\%20DE\%202017.pdf

Puentes de Armas, Tomás, \& Sánchez Valdés, Xiomara. (2019). Las neurociencias para la educación inclusiva en la formación del profesional de la educación infantil. Mendive. Revista de Educación, 17(3), 333-345. Epub 03 de septiembre de 2019. Recuperado en 10 de julio de 2021, http://scielo.sld.cu/scielo.php?script=sci arttext\&pid=S1815-76962019000300333\&lng=es\&tln $\mathrm{g}=\mathrm{es}$. 
Puyo (Bachelor's thesis, Universidad Técnica de Ambato-Facultad de Ciencias de la Salud-Carrera Estimulación Temprana).

Qué es la Neuroeducación. Claves para entenderla e introducirla en el aula (2021)Recuperado de :https://www.educaciontrespuntocero.com/recursos/

que-es-la-neuroeducacion/https://www.aaidd.org/intellectual-disability/definition.

Ramírez Valbuena, W. Á. (2017). La inclusión: una historia de exclusión en el proceso de enseñanza aprendizaje. Cuadernos de Lingüística Hispánica, (30), 211-230. doi: https://doi.org/10.19053/0121053X.n30.0.6195

Rojas, E. G. A., \& González Roque, J. (2017). El trabajo cooperativo como estrategia didáctica para la inclusión en el aula. Revista electrónica de investigación e innovación educativa, 2(1), 38-43.

Rojas-Rojas,C.(2019).Dificultades de aprendizaje en edad escolar. Pensamiento y Acción, núm. 26,(pp.

85

-99)Recuperado

de

https://revistas.uptc.edu.co/index.php/pensamiento accion/article/view/9846/8258

Santana Valencia, E. V. (2019). LA RESILIENCIA EN FAMILIAS QUE VIVEN LA DISCAPACIDAD, DESDE UN ENFOQUE CENTRADO EN LA FAMILIA. Revista Panamericana De Pedagogía, (27). Recuperado a partir de https://revistas.up.edu.mx/RPP/article/view/1682.

Saeed, T. (2011). A Comparative Study of Working Memory in Children with Neurodevelopmental Disorders (Tesis de doctorado). National University of Ireland.

Seda, J. A. (2021). Familias de personas con discapacidad intelectual: ¿Apoyo o sustitución?. Revista Iberoamericana De Bioética, (15), 01-10. https://doi.org/10.14422/rib.i15.y2021.003

Tacca Huamán, D. R., Tacca Huamán, A. L., \& Alva Rodriguez, M. A. (2019). Estrategias neurodidácticas, satisfacción y rendimiento académico en estudiantes universitarios. Cuadernos de investigación educativa, 10(2), 15-32. 
Tillería Pérez, G. D. (2019). El área de educación artística y la discapacidad intelectual: de la teoría de las inteligencias múltiples a la neuroeducación. Homo Sapiens Ediciones. https://elibro.net/es/ereader/poligran/129665?page=75,76.

Vaquerizo, E. J. (2019). Efecto del ejercicio físico en la capacidad cognitiva de escolares durante la educación obligatoria. Lecturas: Educación física y deportes, 24(259), 96-106.Valladolid Recuperado de : http://uvadoc.uva.es/bitstream/handle/

10324/27297/TFM-G749.pdf?sequence=1\&isAllowed=y

Verdugo Alonso, M. Á., Córdoba Andrade, L. y Rodríguez Aguilella, A. (2020). Calidad de vida en familias de personas con discapacidad. Pensando Psicología, 16(1), 1-23.doi:https://doi.org/10.16925/2382-3984.2020.01.01

Villavicencio Aguilar, Carmita, \& López Larrosa, Silvia. (2017). Presencia de la discapacidad intelectual en la familia, afrontamiento de las madres. Fides et Ratio - Revista de Difusión cultural y científica de la Universidad La Salle en Bolivia, 14(14), 99-112. Recuperado en 10 de agosto de 2021, de http://www.scielo.org.bo/

scielo.php?script=sci_arttext\&pid=S2071-081X2017000200007\&lng=es\&tIng=es.

Viñamagus E. (2018). Pictogramas con estrategias didáctica para mejorar la lecto-escritura. Loja, Ecuador.

Willingham, D. T. (2015). Raising kids who read: What parents and teachers can do. John Wiley \& Sons. 


\section{About the Authors:}

Currículo Autores

\section{PERFIL DOCENTE JESSICA AREVALO PARRA:}

Psicóloga, Especialista en evaluación y diagnóstico neuropsicológico, Magister en Neuropsicología clínica, Estudiante de doctorado en Educación, con una amplia experiencia en el campo de la evaluación, rehabilitación y habilitación neuropsicológica en población infantil, así como en temáticas relacionadas con los trastornos del neurodesarrollo, aprendizaje, educación e inclusión, con amplia experiencia en el campo clínico y educativo. Actualmente vinculada en la Institución Universitaria Politecnico Grancolombiano como docente tiempo completo, en la Coordinación del Programa de Especialización en Neuropsicologia Escolar. Docente de la especialización en cognición y neuroaprendizaje en ambiente escolar de la Universidad Pontificia Bolivariana, y de la especialización y maestría en educación de la Universidad la Grancolombia.Adelanta investigaciones relacionadas con habilidades académicas, diseño de instrumentos de evaluación e intervención Neuropsicología con realidad virtual, Neuroeducación en el aula y Neurotecnología.

\section{DANIELA GRAIÑO}

Profesora de Educación Preescolar con más de 20 años de experiencia docente en el ámbito estatal y privado en Buenos Aires Argentina.

Psicóloga

Especialista en Neuropsicología y Rehabilitación cognitiva de la Universidad Favaloro .

Especialista en Métodos de Evaluación Neuropsicológica de la Universidad Favaloro.

Especialista en Neuropsicología Escolar del Politécnico Grancolombiano.

Actualmente vinculada a cursos en Ineco Argentina sobre Teleneuropsicologia. 


\section{SILVIA GONZALEZ}

Psicóloga con excelente formación en el campo educativo y en el campo de investigación, amplia experiencia en el campo organizacional y educativo. Especialista en Neuropsicología escolar, con experiencia como acompañante terapéutica dentro del aula escolar en población infantil y adolescente diagnosticada con trastornos del neurodesarrollo.

Asistente de Investigación en un proyecto de investigación Aplicada asociado a la Institución Universitaria Politécnico Grancolombiano denominado: “Estrategia de intervención para construir cultura tributaria y contable a partir del Monotributo a comerciantes al por menor de tiendas y peluquerías en la zona comercial del barrio Chapinero Norte", así mismo asistente de Investigación de la Institución Universitaria Politécnico Grancolombiano, cuya función consistía en realizar el seguimiento a los diferentes proyectos de investigación de docentes asociados a la Institución Universitaria Politécnico Gancolombiano.

\section{MÓNICA ALEJANDRA MACHADO}

Docente, Psicóloga con énfasis clínico y de la salud, especializada en diagnóstico, evaluación e intervención psicopedagógica y neuropsicológica de las necesidades educativas del aprendizaje y de trastornos del neurodesarrollo de la Institución Universitaria Politécnico Grancolombiano. Con amplia experiencia en el campo clínico y educativo: asesoramiento, orientación y formación de hábitos, pautas de crianza y fortalecimiento en el proceso cognitivo y psicosocial con el sistema educativo y familia.

\section{MILENA FLÓREZ BAUTISTA}

Psicóloga, especialista en neuropsicología escolar, con experiencia en el sector educativo y el área clínica con población infantil. Actualmente vinculada como orientadora escolar en el Colegio Salesiano Juan del Rizzo 